\title{
The American Phytopathological Society Elected and Appointed Officers, Representatives, and Committees for 1999-2000
}

Please note: The most current board and committee rosters are found on-line at APSnet

\section{Council}

President: N. K. Van Alfen

President-Elect: S. A. Slack

Immediate Past President: C. E. Windels

Vice President: N. T. Keen

Secretary: D. J. Jardine

Treasurer: J. L. Sherwood

Editor-in-Chief, Phytopathology: B. I. Hillman

Editor-in-Chief, Plant Disease: G. E. Shaner

Editor-in-Chief, Molecular Plant-Microbe Interactions : J. E. Leach

Editor-in-Chief, Phytopathology News: R. F. Nyvall

Editor-in-Chief, APS Press: S. M. Douglas

Senior Councilor-at-Large: J. E. Loper

Intermediate Councilor-at-Large: R. C. Gergerich

Junior Councilor-at-Large: S. S. Hurtt

Councilor of the Caribbean Division: S. Cantrell

Councilor of the North Central Division: F. W. Nutter Jr.

Councilor of the Northeastern Division: T. J. Burr

Councilor of the Pacific Division: G. A. Chastagner

Councilor of the Potomac Division: E. L. Stromberg

Councilor of the Southern Division: D. T. Beadle

APS Foundation Representative: S. M. Coakley (ex officio)

Executive Vice President: S. C. Nelson (ex officio)

Staff

Executive Vice President/Publisher: Steven C. Nelson

\section{Representatives}

American Association for the Advancement of Science: J. Cook

American Institute of Biological Sciences: (to be determined)

American National Standards Institute: J. E. Elson

American Type Culture Collection: R. S. Nelson

Coalition for Education in the Life Sciences: M. A. Sulzinski

Coalition for Funding Agricultural Research: S. A. Tolin

Coalition for Research on Plant Systems: S. A. Tolin

Council for Agricultural Science and Technology: B. J. Jacobsen

International Commission on Microbial Ecology: S. Lindow

International Society of Arboriculture: R. J. Stipes

International Society for Plant Pathology: Chair: A. M. Alvarez. G. S. Abawi, L.

V. Madden, J. Moyer, C. C. Mundt, B. W. Pennypacker, L. Sequeira, P. S.

Teng, A. H. van Bruggen, R. S. Zeigler

International Union of Microbiological Societies: D. C. Gross

Mycological Society of America: C. M. Stiles

National Committee on Worker Safety Standards: S. A. Alexander

National Institutes for the Environment: T. A. Kucharek

National Research Council, Commission on Life Sciences: D. J. Jardine

National Research Council, CLS/Board of Agriculture: S. A. Slack

Society of Environmental Toxicology and Chemistry: M. Simini

Society of Nematologists: E. L. Davis

APS Historian

P. D. Peterson, Jr.

APS Foundation, Board of Directors

Chair: S. M. Coakley. Vice Chair: B. J. Jacobsen. Secretary: A. O. Paulus. Treasurer:

J. R. French. Past Chair: O. W. Barnett. M. N. Cline, D. A. Cooksey, T. K.

Mitchell (ex officio), S. C. Nelson (ex officio), J. L. Sherwood (ex officio)

\section{Office of International Programs Advisory Board}

Director: R. A. Bennett. Immediate Past Director: L. Sequeira. G. S. Abawi, K. S. Cardwell, S. C. Nelson (APS Executive Vice President), A. O. Paulus(APS Foundation Representative), N. W. Schaad, J. R. Steadman

Education: Chair: K. S. Cardwell. G. S. Abawi, H. J. Dubin, J. H. McBeath

Policies and Program(s): Chair: G. S. Abawi. Vice Chair: H. J. Dubin. L. Black,

R. F. Line, R. C. Ploetz, E. B. Smalley, J. R. Steadman, Z. Xiong

Research: Chair: N. W. Schaad. S. H. De Boer, G. A. Forbes, R. F. Line, R. C. Ploetz Service: Chair: J. R. Steadman. B. Brown, S. H. De Boer, H. A. Lamey, G. A. Zentmyer

Publication no. P-1999-1130-020
National Plant Pathology Board

Chair: A. K. Vidaver. J. Amador, O. W. Barnett, R. J. Cook, D. E. Mathre, L. J. Stowell, S. A. Tolin

Office of Electronic Communication (OEC)

Director: J. D. MacDonald. A. M. Alvarez, C. L. Ash (ex officio), S. M. Douglas, D. C. Gross, G. G. Grove, R. C. Ploetz, G. L. Schumann, M. Wimer (ex officio)

OEC Electronic Technology Advisory Committee: Chair: D. M. Eastburn. Vice Chair: N. Grunwald. Immediate Past Chair: C. T. Bull. A. R. Biggs, G. G. Grove, D. B. Marti, G. W. Moorman, T. D. Murray

APS Publications Board

Chair: G. E. Shaner. S. M. Douglas, B. I. Hillman, J. E. Leach, J. D. MacDonald, R. F. Nyvall, M. Wimer (ex officio)

OPAE Advisory Board

Director: G. C. Bergstrom. D. A. Albaugh, C. L. Ash (ex officio), M. J. Bjerkness (ex officio), J. E. Carroll, M. N. Cline, M. A. Ellis, D. C. Gross, A. L. Hope (ex officio), B. D. Mogen, S. C. Nelson (ex officio), M. L. Powelson, J. R. Steadman, N. K. Van Alfen, A. K. Vidaver

Communications: Chair: D. A. Albaugh. C. L. Ash (ex officio), M. J. Bjerkness (ex officio), R. H. Brlansky, L. D. Carsten, M. N. Cline, R. F. Line, R. F. Nyvall (ex officio), N. W. Schaad, P. R. Sellers, M. D. Steinberg

Editors of APS Press

Editor-in-Chief: S. M. Douglas. Editor-in-Chief-Elect: R. C. Ploetz. Senior Editors: D. M. Benson, A. Bergamin Filho, E. J. Braun, W. Chun, M. L. Daughtrey, T. R. Gottwald, A. B. Gould, R. Loria, K.-B. G. Scholthof

Editors of BIOLOGICAL AND CULTURAL TESTS FOR CONTROL OF PLANT DISEASES

Editor: M. T. McGrath. Section Editors: M. J. Boehm, K. L. Bowen, D. R. Cooley, G. J. Holmes, T. S. Isakeit, D. J. Jardine, A. P. Keinath, P. E. Lipps, G. P. Munkvold, J. Williams-Woodward

Editors of MOLECULAR PLANT-MICROBE INTERACTIONS

Editor-in-Chief: J. E. Leach. Senior Editors: J. L. Dangl, M. J. Daniels, M. J. Harrison, S. Lindow, A. Osbourn, P. F. Palukaitis, H. P. Spaink, V. M. Williamson. Associate Editors: M. Arlat, F. J. Barras, D. L. Beck, C. L. Bender, N. J. Brewin, J. P. Carr, X. Dong, D. J. Ebbole, F. Garcia-Arenal, G. Gheysen, J. R. Glazebrook, F. Govers, M. Grant, A. M. Hirsch, M. M. Holsters, W. H. Knogge, J. W. Kronstad, B. N. Kunkel, S. A. Lommel, K. W. Mendgen, T. Nuenberger, J. E. Parker, S. Perotto, L. S. Pierson III, J. M. Salmeron, F. Sánchez, D. Scheel, J. E. Schoelz, J. Stougaard, J. Sweigard, N. D. Young

\section{Editors of PHYTOPATHOLOGY}

Editor-in-Chief: B. I. Hillman. Senior Editors: D. M. Bird, D. A. Cuppels, R. L. Gilbertson, S. H. Hulbert, A. M. Jarosz, M. Mazzola, R. S. Nelson, K. V. Subbarao, X.-B. Yang. Associate Editors: D. Andrivon, J. K. Brown, M. J. Davis, J. A. Duthie, K. S. Elias, D. W. Gabriel, S. E. Gold, T. R. Gordon, N. H. Grimsley, J. Hammond, Y. Jin, D. Y. Kobayashi, R. P. Larkin, M. G. Milgroom, C. C. Mundt, T. L. Peever, N. B. Quigley, J. M. Raaijmakers, M. J. Roossinck, C. L. Schardl, D. A. Schisler, S. M. Schneider, H. B. Scholthof, J. E. Sherwood, L. C. van Loon

Editors of PLANT DISEASE

Editor-in-Chief: G. E. Shaner. Senior Editors: A. R. Biggs, J. C. Correll, L. J. Francl, D. L. Hopkins, S. A. Miller, M. E. Ostry, J. K. Pataky, C. M. Rush, H. D. Shew, H. E. Waterworth. Feature Editor: M. L. Gleason. Disease Notes Assigning Editor: R. M. Davis. Focus Editor: T. Kommedahl. Associate Editors: B. D. Bruton, P. D. Colyer, D. R. Cooley, B. M. Cunfer, L. L. Domier, L. J. du Toit, K. C. Eastwell, S. A. Enebak, R. D. Gitaitis, G. G. Grove, R. W. Hammond, T. C. Harrington, D. E. Hershman, R. M. Hunger, J. S. Lehman, P. S. McManus, W. T. Nishijima, J. P. Noe, J. S. Russin, D. A. Samac, H. Scherm, K. L. Stevenson, J. P. Wilson, C. P. Woloshuk

Society Internal Relations

Awards and Honors: Chair: J. H. Hill. Vice Chair: I. Barash. Immediate Past Chair: S. Lindow. T. J. Burr, D. I. Mills, G. Payne, W. B. Stevenson Committee on Committees: Chair: J. E. Loper. R. C. Gergerich, S. S. Hurtt Executive: Chair: N. K. Van Alfen. D. J. Jardine, N. T. Keen, J. E. Loper, S. C. Nelson, J. L. Sherwood, S. A. Slack, C. E. Windels 
Financial Advisory: Chair: J. L. Sherwood. S. M. Douglas, N. T. Keen, J. E. Loper, S. C. Nelson (ex officio), S. A. Slack, E. L. Stromberg, N. K. Van Alfen (ex officio)

Headquarters Operations: S. C. Nelson (ex officio), J. L. Sherwood, S. A. Slack, N. K. Van Alfen, C. E. Windels, AACC representatives

Meeting Site Selection: Chair: R. C. Gergerich. D. T. Beadle, T. J. Burr, G. A. Chastagner, S. S. Hurtt, F. Labatt (ex officio), J. E. Loper, F. W. Nutter Jr., E. L. Stromberg

Nominating: Chair: R. C. Gergerich. D. T. Beadle, T. J. Burr, G. A. Chastagner, F. W. Nutter Jr., E. L. Stromberg

Placement: Chair: D. A. Palmer. Vice Chair: E. A. Sobek. Immediate Past Chair: R. A. Kuznia. C. H. Bock, P. J. Cotty, L. J. du Toit, P. M. McCabe, S. L. Walker

Program: Chair: S. A. Slack. Vice Chair: N. T. Keen. Immediate Past Chair: N. K. Van Alfen. J. E. Loper, S. C. Nelson, Committee Chairs

Sustaining Associates: Chair: M. N. Cline. Immediate Past Chair: A. B. Bassi. P. F. Colbaugh, G. W. Moorman, S. R. Siemer

\section{Society General Policies}

Biotechnology Impact Assessment: Chair: J. L. Norelli. Vice Chair: B. K. Duffy. Immediate Past Chair: A. L. Pilgeram. J. H. Andrews, G. K. Batra, G. J. Defago, M. R. Finkh, D. C. Thompson

Collections and Germ Plasm: Chair: R. F. Line. Vice Chair: J. C. Rupe. Vice Chair-Elect: M. E. Miller. Immediate Past Chair: J. A. Thies. J. A. Foster, A. G. Gillaspie, J. H. Hill, S. Leath, B. Lovic, M. E. Meadows, A. Y. Rossman, H. F. Schwartz, C. E. Thomas, A. T. Tschanz

Cultural Diversity: Chair: A. E. Dorrance. Vice Chair: M. A. Morant. Immediate Past Chair: W. Miller. M. T. Abdullah, P. D. Adams, D. M. Huber, J. E. Lundquist, N. Ntahimpera, J. J. Roberts, B. P. Singh, R. H. von Qualen

Extension: Chair: M. A. Ellis. Vice Chair: M. A. Draper. Immediate Past Chair: P. R. Sellers. E. A. Brown, R. L. Forster, N. P. Goldberg, D. E. Hershman, S. A. Johnston, S. R. Koenning, J. S. Lamboy, L. P. Pottorff, R. L. Schlub, C. A. Smith

Graduate Student: Chair: T. K. Mitchell. Vice Chair: A. E. Glenn. Immediate Past Chair: J. S. Batten. M. G. Anderson, V. M. Aris, C. A. Bradley, L. R. Emele, M. Goellner, D. B. Langston, C. Riegel, S. J. Scheuerell, P. M. Sforza

Industry: Chair: E. C. Tedford. Vice Chair: C. T. Schiller. Immediate Past Chair: J. L. Riggs. N. B. Carroll, S. R. Foor, B. J. Jacobsen, C. S. Morton, C. L. Palmer, R. E. Ross, K. W. Seebold, K. K. Shetty

Industry Advisory: Chair: N. B. Carroll. C. M. Becker, M. N. Cline, S. P. Falk, J. R. James, A. J. Julis, R. P. Kaiser, D. F. Kendra, K. T. Kmetz, H. V. Morton, J. L. Riggs, B. J. Shortt, C. L. Sutula

Phytopathology News Advisory: C. L. Ash, R. C. Gergerich, S. S. Hurtt, J. E. Loper, R. P. Mulrooney, G. L. Schumann, M. Wimer

Private Practice: Chair: W. R. Landis. Vice Chair: C. H. Mellinger. Vice Chair-Elect: W. T. Cobb. Immediate Past Chair: J. H. Hart. G. Bonn, S. J. Day, D. D. Miller

Regulatory Plant Pathology: Chair: R. E. Ykema. Vice Chair: L. G. Brown. Immediate Past Chair: C. L. Schoulties. S. D. Cohen, J. H. Ghazanfari, K. L. Kosta, A. Mengistu, G. M. Milbrath, A. B. Sinderman, A. T. Tschanz, X.-B. Yang

Teaching: Chair: T. A. Evans. Vice Chair: M. B. Riley. Immediate Past Chair: B. D. Mogen. D. N. Appel, R. B. Carroll, J. H. Connolly, C. R. Curtis, K. D. Gwinn, M. A. Langham, J. C. Locke, A. T. Saad, E. L. Stewart, M. A. Sulzinski, P. Vincelli

Women in Plant Pathology: Chair: N. Burelle. Vice Chair: L. E. Hanson. Immediate Past Chair: K. L. Everts. M. T. Abdullah, D. B. Lopes, L. Ortiz, L. G. Skoglund, M. Smither-Kopperl, J. M. Windes, N. K. Zidack

Youth Program: Chair: C. R. Curtis. Vice Chair: R. N. Raid. Immediate Past Chair: C. H. Walkinshaw. A. J. Anderson, J. J. Choi, K. E. Duncan, J. Esnard, D. W. Kalb, J. M. Krupinsky, L. D. Moore, B. L. Randall-Schadel, A. Tally, P. W. Tooley

\section{Subject Matter}

Bacteriology: Chair: L. S. Pierson III. Vice Chair: F. J. Louws. Immediate Past Chair: W. F. Mahaffee. J. D. Barak, G. A. Beattie, S. B. Beck Von Bodman, J. C. Chen, R. Frederick, S. A. Miller, S. Q. Pan, A. R. Poplawsky, K. L. Schroeder, G. W. Sundin

Biochemistry, Physiology and Molecular Biology: Chair: C. P. Woloshuk. Vice Chair: R. G. Upchurch. Immediate Past Chair: P. A. Gay. S. B. Beck Von Bodman, J. P. Bolar, K. R. Davis, M. B. Dickman, R. Hammerschmidt, H. Leung, S. W. Meinhardt, T. J. Wolpert, M. N. Zook

Biological Control: Chair: V. O. Stockwell. Vice Chair: C. M. Press. Immediate Past Chair: L. L. Kinkel. D. Gurian-Sherman, C. L. Hartman, D. M. Huber, J. L. Parke, E. N. Rosskopf, D. C. Sands, S. F. Shamoun, W. F. Stoneman, D. O. Te Beest, R. S. Utkhede, P. Warrior

Chemical Control: Chair: L. E. Zang. Vice Chair: N. Lalancette. Immediate Past Chair: D. C. Thompson. D. T. Beadle, T. B. Brenneman, A. R. Chase, R. Gold, C. L. Kohls, T. A. Melton, T. M. Momol, G. Olaya

Diagnostics: Chair: T. C. Stebbins. Vice Chair: K. K. Rane. Immediate Past Chair: E. J. Hudgins. J. W. Beale, S. T. Koike, S. M. Lerner, J. Mullen, B. Rao, K. L. Sirois, M. A. Williamson

Diseases of Ornamental Plants: Chair: J. C. Locke. Vice Chair: A. B. Sinderman. Immediate Past Chair: D. L. Clement. M. J. Klopmeyer, T. M. Likens, R. J. McGovern, S. R. Vann, J. E. Watkins
Environmental Quality and Plant Health: Chair: M. Simini. Vice Chair: P. A. Gay. Immediate Past Chair: J. R. Glazener. R. Bulluck, C. R. Curtis, D. M. Karasevicz, J. Kotcon, M. S. Krause, J. S. Lamboy, M. Skaria, V. L. Smith

Epidemiology: Chair: C. S. Thomas. Vice Chair: W. F. Pfender. Immediate Past Chair: N. Grunwald. S. Chakraborty, K. M. Emery, N. Evans, G. A. Forbes, J.J. Hao, C. Hong, F. W. Nutter Jr., S. Sanogo, H. Su

Forest Pathology: Chair: K. O. Britton. Vice Chair: W. J. Otrosina. Immediate Past Chair: D. R. Bergdahl. D. Bannwart, L. Bernier, S. D. Cohen, S. A. Enebak, R. C. Hamelin, L. M. Haugen, J. L. Kerrigan, J. T. Kliejunas, W.-H. Ko, P. C. Spaine, J. A. Walla, P. J. Zambino

Genetics: Chair: D. M. Francis. Vice Chair: T. L. Peever. Immediate Past Chair: U. L. Rosewich. W. Chen, S. E. Gold, S. Kang, G. Kuldau, F. N. Lee, C. M. Liddell, R. J. Nelson, T. Plyer, J. B. Rasmussen

Host Resistance: Chair: J. M. Windes. Vice Chair: J. A. Thies. Immediate Past Chair: J. L. Starr. X. Chen, K. L. Crabtree, A. K. Culbreath, A. E. Dorrance, P. T. Himmel, G. L. Tylka, W. L. Wiebe, J. P. Wilson, I. E. Yates, T. A. Zitter

Integrated Pest Management: Chair: G. L. Schuster. Vice Chair: R. D. Cartwright. Immediate Past Chair: H. V. Morton. S. A. Alexander, E. E. Bauske, D. S. Egel, A. P. Keinath, D. E. Legard, B. E. Paulrud, G. S. Smith, E. L. Stromberg, N. Zriba Mycology: Chair: F. M. Dugan. Vice Chair: M. A. Cubeta. Immediate Past Chair: P. V. Oudemans. R. E. Baird, D. M. Beyer, H. C. Kistler, D. J. Royse, C. M. Stiles, L. J. Vaillancourt

Mycotoxicology: Chair: F. Trail. Vice Chair: G. P. Munkvold. Immediate Past Chair: P. J. Cotty. H. K. Abbas, C. W. Bacon, K. S. Cardwell, K. E. Damann, A. E. Desjardins, J. P. Duvick, N. J. Keller, M. P. McMullen, D. M. Wilson, C. P. Woloshuk

Nematology: Chair: J. A. LaMondia. Vice Chair: C. S. Johnson. Immediate Past Chair: L. A. Payan. T. J. Baum, J. O. Becker, W. T. Crow, D. W. Dickson, J. Esnard, D. Neher, T. L. Niblack, R. K. Niles, R. D. Riggs, E. J. Sikora, B. P. Singh

Pathogen Resistance: Chair: G. Olaya. Vice Chair: P. I. Lewis. Immediate Past Chair: W. D. Koeller. T. B. Brenneman, K. L. Crabtree, S. N. Jeffers, G. M. Leavitt, P. S. McManus, R. N. Raid, R. A. Romero, F. P. Wong, H. L. Ypema

Phyllosphere Microbiology: Chair: H. C. Hoch. Vice Chair: J. Mercier. Immediate Past Chair: P. S. McManus. D. Aylor, M. Brandl, J. C. Hubbard, K. B. Johnson, O. Kilic, L. L. Kinkel, S. Lindow, C. C. Mundt, R. L. Nicholson, V. O. Stockwell

Plant Disease Detection: Chair: R. R. Martin. Vice Chair: L. Levy. Immediate Past Chair: J. C. Rupe. V. DeHerrera, S. N. Jeffers, P. D. Kharbanda, C. A. Levesque, W. H. Sheta, M. G. Tiffany

Plant Disease Losses: Chair: F. W. Nutter Jr. Vice Chair: S. Chakraborty. Immediate Past Chair: M. H. Royer. J. C. Comstock, C. A. Hollier, D. L. Long, Y. Luo, W. W. Turechek, A. Wrather, C. L. Xiao

Postharvest Pathology: Chair: J. A. Bartz. Vice Chair: W. S. Conway. Immediate Past Chair: J. A. Bartz. B. D. Bruton, K. S. Cardwell, G. J. Holmes, W. J. Janisiewicz, D. F. Kendra, G. P. Munkvold, P. L. Sholberg, J. L. Smilanick

Seed Pathology: Chair: A. M. Alvarez. Vice Chair: C. J. Kurowski. Immediate Past Chair: W. L. Wiebe. G. C. Bergstrom, H. Bouzar, G. P. Hoyos, J. W. Lorbeer, B. Lovic, L. M. Shepherd, A. B. Thornton, M. G. Tiffany, A. M. VanBuren, R. R. Walcott

Soil Microbiology and Root Diseases: Chair: K. V. Subbarao. Vice Chair: D. R. Fravel. Immediate Past Chair: B. K. Duffy. R. G. Bhat, W. Chen, J. A. GraciaGarza, R. D. Martyn, S. L. Meyer, K. K. Shetty, N. Shishkoff, M. L. SmitherKopperl, A. G. Stone

Tropical Plant Pathology: Chair: J. Y. Uchida. Vice Chair: R. G. McGuire. Immediate Past Chair: M. J. Davis. A. M. Alvarez, S. J. Bagenski, R. Bandyopadhyay, J. C. Comstock, D. R. Greenough, R. F. Lee, A. A. MacNab

Turfgrass Pathology: Chair: E. B. Nelson. Vice Chair: M. J. Boehm. B. B. Clarke, D. Green, K. J. Langan, R. X. Latin, E. A. Milus, J. F. Powell, G. L. Schumann, L. J. Stowell, J. M. Vargas

Virology: Chair: K. M. Scheets. Vice Chair: W. M. Wintermantel. Immediate Past Chair: J. N. Culver. V. Bowman-Vance, J. K. Brown, A. L. Eggenberger, D. T. Gordon, R. Grumet, J. F. Murphy, K. L. Perry, C. M. Rush, H. B. Scholthof, G. C. Wisler, M. Zaitlin

\section{APS Press Committees}

Illustrations of Plant Pathogens and Diseases: Chair: E. J. Braun. Immediate Past Chair: G. L. Schumann. G. J. Holmes, G. W. Hudler, V. Kumar, G. W. Moorman, N. R. O’Neill, J. E. Partridge, A. C. Schilder, G. R. Stanosz, T. J. Volk, J. E. Watkins

Phytopathological Classics: Chair: P. D. Peterson. Immediate Past Chair: T. D. Murray. A. Bergamin Filho, D. W. Fulbright, C. M. Liddell, K.-B. G. Scholthof

Standardization of Common Names of Plant Diseases: Chair: M. Putnam. Vice Chair: P. F. Bertrand. Immediate Past Chair: B. L. Teviotdale. C. W. Averre, J. H. Hart, M. E. Matheron, D. M. Rizzo, J. L. W. Woodward, T. A. Zitter

New Fungicide and Nematicide Tests: Chair: P. Vincelli. Vice Chair: D. A. Rosenberger. Immediate Past Chair: R. E. Baird. Editor: R. N. Raid. G. T. Berggren, B. A. Fulling, T. L. Kirkpatrick, C. S. Kousik, A. A. MacNab, B. S. Sipes, T. B. Sutton, L. W. Timmer, W. F. Wilcox 